\title{
Simultaneity and sequence in the perception of apparent motion
}

\author{
LEE A. GILROY \\ Middle Tennessee State University, Murfreesboro, Tennessee \\ AND \\ HOWARD S. HOCK \\ Florida Atlantic University, Boca Raton, Florida
}

\begin{abstract}
Motion perception usually is accompanied by the phenomenological impression of sequence as objects move through successions of locations. Nonetheless, there is accumulating evidence that sequential information is neither necessary nor sufficient for perceiving motion. It is shown here that apparent motion is specified by counterchange rather than sequence - that is, by co-occurring toward- and away-from-background changes at two spatial locations, regardless of whether the changes are simultaneous or sequential. Motion is perceived from the location of the toward to the location of the away change, even when the changes occur in reverse temporal order. It is not perceived for sequences of away or toward changes, as would be expected if motion were specified by onset or offset asynchronicity. Results previously attributed to onset and offset asynchrony are instead attributable to onsets and offsets occurring in close temporal proximity at the same location. This was consistent with units for detecting away and toward changes that are temporally biphasic; that is, they are excited by changes in one direction and inhibited by immediately preceding or immediately following changes in the opposite direction. These results are accounted for by a model for counterchange-specified motion entailing the biphasic detection of toward and away changes.
\end{abstract}

When an object moves through space, it occupies a series of spatial locations along its trajectory, providing a strong phenomenological impression of sequence. It is perhaps for this reason that motion-specifying stimulus information has been thought to be sequential. Accordingly, classical models have emphasized the detection of sequential information for pairs of visual events, incorporating temporal delays in order to bring the activations produced by the events into temporal coincidence (e.g., Reichardt, 1961). Consistent with motion being specified by sequences of stimulus events, experimental researchers have provided evidence for effects of stimulus onset asynchrony (the time between the onsets of successive events) and interstimulus interval (the time between the offset of one event and the onset of the next event) on the perception of apparent motion (Burt \& Sperling, 1981; Kahneman \& Wolman, 1970; Kolers, 1964; Korte, 1915; Lakatos \& Shepard, 1997; Wertheimer, 1912).

The present article furthers our investigation of an alternative to sequence-determined motion perception: namely, that motion can be specified by a pattern of stimulus change (Gibson, 1968) entailing counterchangethat is, oppositely signed changes in luminance or texture contrast at two locations. The experiments distinguish between counterchange and sequential information and provide further evidence that motion energy detection is not responsible for the perception of single-element apparent motion between pairs of simultaneously visible surfaces (motion energy extraction is not precluded as the basis for motion perception under other circumstances).

In standard apparent motion (Figure 1A), a surface is discontinuously displaced from one location to another; so, conceivably, motion could be specified by the sequence of locations that the surface occupies. However, the displacement also creates counterchange. For lighterthan-background surfaces, luminance decreases to the background value at the location initially occupied by the surface and increases away from the background value at the location newly occupied by the displaced surface. In generalized apparent motion (Figures 1B and 1C), the surface is simultaneously visible at both of its locations, but the pattern of toward and away changes is the same (Hock, Kogan, \& Espinoza, 1997; Johansson, 1950). Surfaces do not change location for these stimuli, so the perception of apparent motion does not require detecting a sequence of location changes. Exner (1875) similarly showed that apparent motion can be perceived for successive flashes that are too close to each other to be spatially resolved.

Counterchange-specified motion is determined by background-relative stimulus changes that can be simultaneous rather than sequential. For the stimuli in Figures 1B and 1C, apparent motion potentially can be based on either first-order luminance counterchange or second-order contrast counterchange (the contrast is with

H. S. Hock, hockhs@fau.edu 


\section{A Standard Apparent Motion}

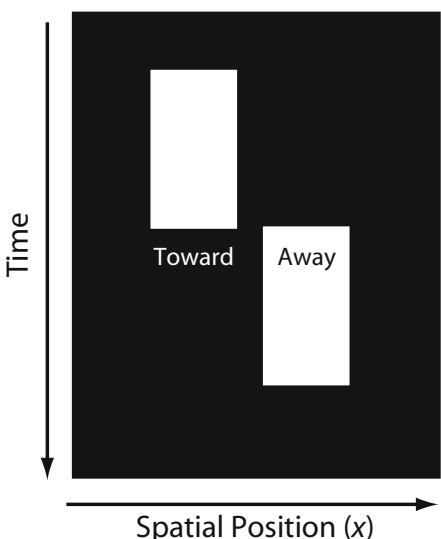

B Generalized Apparent Motion

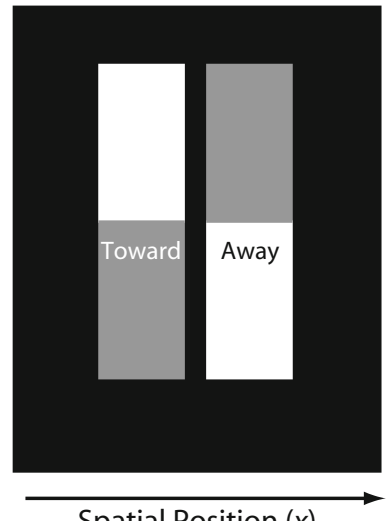

Spatial Position $(x)$
C Generalized Apparent Motion

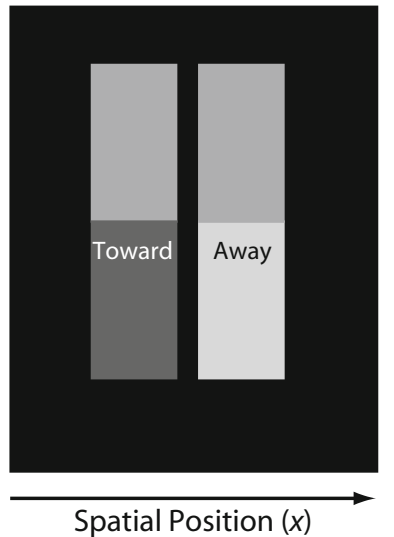

D
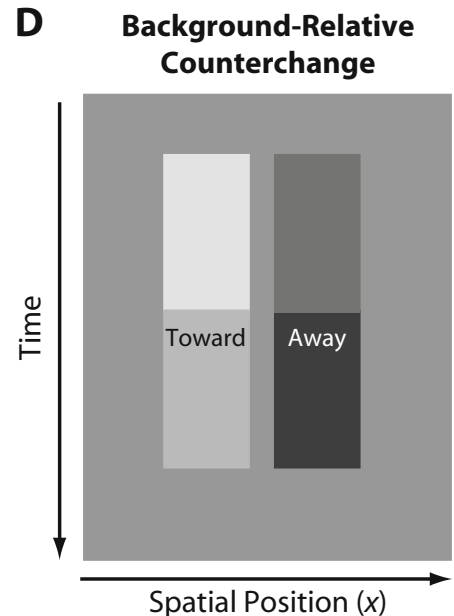

F Rightward Motion EnergyNo Counterchange

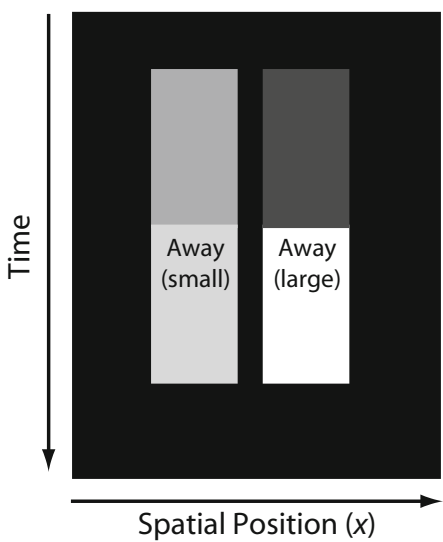

E Counterchange With Change in Global Luminance

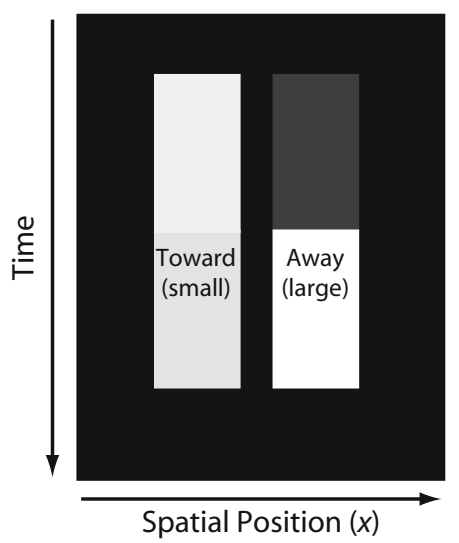

G Higher Luminance Contrast Always on the Right

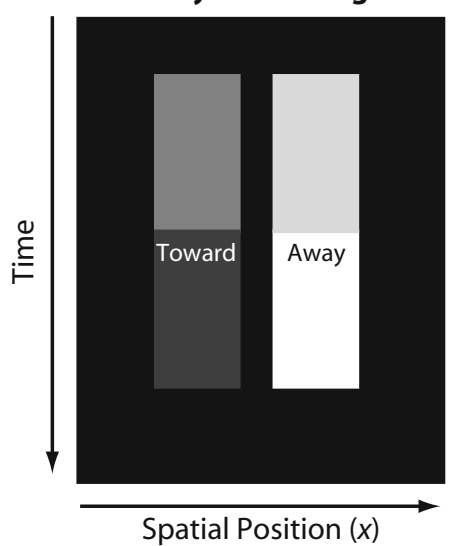

Figure 1. Graphical representations of the discontinuous luminance changes produced by (A) a standard apparent motion stimulus, (B) a generalized apparent motion stimulus, $(C)$ a generalized apparent motion stimulus for which the luminance values of the surfaces initially are the same, eliminating the possibility that motion perception is based on sequential changes in one direction for high-contrast surfaces or sequential changes in the opposite direction for low-contrast surfaces, (D) a generalized apparent motion stimulus for which there is background-relative counterchange, even though the luminance simultaneously decreases at both surface locations, (E) a generalized apparent motion stimulus for which counterchange-specified motion is perceived, despite the average luminance of the surfaces increasing, (F) a stimulus for which there is rightward motion energy (the lighter of the surfaces shifts from the left to the right) but motion is not perceived because there is no counterchange, and (G) a generalized apparent motion stimulus for which counterchange-specified motion is perceived despite the lighter surface always being on the right, so there are neither trackable displacements nor trackable changes in the location with the higher luminance. 
respect to each surface's background). ${ }^{1}$ For the stimulus in Figure 1D, however, there is only second-order contrast counterchange. When both surfaces decrease in luminance, there is a toward-background decrease in contrast for the lighter-than-background surface and an awayfrom-background increase in contrast for the darker-thanbackground surface. Motion begins at the toward change and ends at the away change, even when there are large frame-to-frame changes in globally averaged luminance, as in Figure 1E.

Hock, Gilroy, and Harnett (2002), Gilroy and Hock (2004a), and Hock and Gilroy (2005) have shown that counterchange detection is the basis for perceiving singleelement apparent motion between pairs of simultaneously visible surfaces that either are uniform in luminance or are textured (checkerboards). Motion was perceived for these stimuli when the motion-specifying stimulus attributes simultaneously changed toward and away from the background, eliminating sequential information as a factor. ${ }^{2}$ The results thus join others in demonstrating that sequential change is not necessary for the perception of apparent motion. For example, Mather (1984) found that replacing a luminance-defined edge by a blank field (and vice versa) could result in motion perception, and Johansson (1950), Hock et al. (1997), and Lappin, Tadin, and Whittier (2002) have shown that motion can be perceived when luminance simultaneously changes in opposite directions for pairs of visible surfaces. Simultaneous change results in motion perception for pairs of luminance-defined surfaces with opposite luminance polarity (Anstis \& Mather, 1985; Hock et al., 2002), pairs of sine gratings (Watson, 1986), and pairs of textured surfaces (Mather \& Anstis, 1995).

\section{Motion Energy Detection}

The possibility that motion energy (Adelson \& Bergen, 1985) rather than counterchange is the basis for motion between two simultaneously visible surfaces was tested with stimuli for which the luminance (and background-relative contrast) of one surface initially was greater than that of the other but both surfaces increased unequally, so that luminance and luminance contrast became greater for what initially was the surface with the lower luminance and luminance contrast, as in Figure 1F (Hock et al., 2002). Motion was not perceived even though first- and second-order motion energy was generated by this shift in the location with the higher luminance and luminance contrast. This was because there was no first- or second-order counterchange: Luminance and luminance contrast increased for both surfaces rather than decreasing for one surface and increasing for the other. (Pairs of stimuli with and without counterchange were matched in motion energy content by integrating and comparing the energy within different quadrants of the Fourier-transformed space-time profiles. Motion was perceived only for the stimuli with counterchange.)

The same observation was made for pairs of simultaneously visible checkerboard surfaces presented against a checkerboard background (Gilroy \& Hock, 2004a). The texture contrast of the checkerboard surfaces increased unequally such that the texture contrast became greater for what initially was the surface with the lower texture contrast. Although second-order motion energy was generated by this shift in the location with the higher texture contrast, motion was not perceived, again because there was no second-order counterchange (i.e., background-relative texture contrast increased for both surfaces rather than decreasing for one surface and increasing for the other). (Pairs of stimuli with and without counterchange were matched in motion energy content by integrating and comparing the energy within different quadrants of the postrectification Fourier-transformed space-time profiles.)

As indicated earlier, the above results do not mean that motion energy detection is not responsible for the motion perceived under other circumstances. It can be the basis for motion perception when counterchange is present (Hock \& Nichols, 2004) and when it is absent (Chubb \& Sperling, 1989). By showing that it is not a factor for the perception of single-element apparent motion between two simultaneously visible surfaces, we establish a window through which counterchange-specified apparent motion can be examined in relative isolation.

\section{Attentive Feature Tracking}

The possibility that the perception of single-element apparent motion requires attentive feature tracking (Cavanagh, 1992) was addressed by Hock et al. (2002) and Gilroy and Hock (2004a) with stimuli for which there was no trackable stimulus information (Figure 1G). They found that counterchange-specified motion was perceived, even though two surfaces were simultaneously visible and there was no change in the location with the greater luminance or texture contrast (e.g., it always is on the right in Figure 1G). In previous studies and in the present study, the possibility of attentive feature tracking was minimized by instructing participants to maintain attention midway between the two surfaces throughout the trial, and to avoid shifting their attention between the surfaces. This does not mean that attentive feature tracking cannot be responsible for motion perception under other circumstances. Instructions encouraging attentive tracking can enhance the perception of motion between pairs of simultaneously visible surfaces for which motion perception otherwise would be relatively weak (Hock et al., 2002).

\section{Counterchange- Versus Sequence-Specified Motion}

In the experiments above, motion resulted from simultaneous changes in luminance and/or texture contrast. We ask in this article whether single-element apparent motion perception can be determined by sequential stimulus information when such information is available. If not a sequence of spatial locations, as is present for standard apparent motion stimuli, can motion perception be based on a sequence of luminance or texture contrast changes at two locations? The reported experiments show that the detection of such sequential changes is neither necessary nor sufficient for the perception of single-element apparent motion and show, instead, that it depends on the detection of counterchange. 


\section{EXPERIMENT 1}

In this experiment, it was determined whether (1) counterchange remains the basis for motion perception between simultaneously visible surfaces when backgroundrelative changes specifying the start and end of the motion path are sequential, (2) motion direction is determined by counterchange or sequence when they specify motion in opposite directions, and (3) the sequential presentation of same signed changes at different spatial locations is sufficient for motion perception.

\section{Method}

Four conditions were created by the orthogonal combination of change direction (toward or away) and temporal order. Counterchange- and sequence-specified motion were in the same direction when a toward change at one location was followed by an away change at the other. They specified motion in opposite directions when an away change at one location was followed by a toward change at the other. There was no counterchange when there was a succession of toward or a succession of away changes at the two locations. If motion were perceived for the latter, it would be consistent with its specification by sequential stimulus information (instructions to participants stressed the avoidance of attentional tracking between the two surfaces). The time between pairs of changes, the interchange interval (ICI), was varied.

Two small squares $(12 \times 12 \mathrm{~min}$ in Experiments 1A and 1B; $34 \times$ 34 min in Experiment 1C) were centered within a rectangular background $\left(3.5^{\circ} \times 2.0^{\circ}\right)$, which in turn was centered within a darkened monitor (luminance $<0.001 \mathrm{~cd} / \mathrm{m}^{2}$ ). The center-to-center separation of the square surfaces was $72 \mathrm{~min}$ in Experiments $1 \mathrm{~A}$ and $1 \mathrm{~B}$ and $103 \mathrm{~min}$ in Experiment 1C. A pair of $6 \times 12 \mathrm{~min}$ fixation lines (luminance $=33.0 \mathrm{~cd} / \mathrm{m}^{2}$ ) was presented at the middle-top and middlebottom of the rectangular background. A chinrest maintained viewing distance at $35.8 \mathrm{~cm}$ in Experiments $1 \mathrm{~A}$ and $1 \mathrm{~B}$ and at $91.4 \mathrm{~cm}$ in Experiment 1C. There were three frames per trial. The durations of Frames 1 and 3 were fixed at $2 \mathrm{sec}$. The Frame 2 duration (the ICI) ranged from 0 to 600,0 to 400 , or 0 to $120 \mathrm{msec}$ (Frame 2 was omitted when the ICI was 0 ). The temporal structure is illustrated in Figure 2.

Experiment 1A. Both surfaces were lighter than the background, as in Figures 2A-2D. The Frame 1 luminance of both surfaces was $40.0 \mathrm{~cd} / \mathrm{m}^{2}$. During Frame 2, it decreased to $19.5 \mathrm{~cd} / \mathrm{m}^{2}$ or increased to $94.0 \mathrm{~cd} / \mathrm{m}^{2}$ for one surface, and during Frame 3, it increased to $94.0 \mathrm{~cd} / \mathrm{m}^{2}$ or decreased to $19.5 \mathrm{~cd} / \mathrm{m}^{2}$ for the other surface. ${ }^{3}$ Luminance changes were matched with respect to background-relative luminance change (BRLC), a metric shown to account for the frequency of motion perception for generalized apparent motion stimuli (Hock et al., 1997). The BRLC for each surface, 0.9, was the change in its luminance divided by the difference between the mean of its two luminance values and the luminance of the background $\left(6.9 \mathrm{~cd} / \mathrm{m}^{2}\right)$. There were two blocks of 112 trials during each of three testing sessions, with seven ICI order-randomized values ranging from 0 to $600 \mathrm{msec}$ in one block and from 0 to $120 \mathrm{msec}$ in the other.

Experiment 1B. One surface was lighter and the other darker than the background $\left(41.0 \mathrm{~cd} / \mathrm{m}^{2}\right.$, as in Figures $2 \mathrm{E}$ and $\left.2 \mathrm{~F}\right)$. When luminance increased (or decreased) for both surfaces, there was a toward-background contrast change for one surface and an awayfrom-background contrast change for the other surface. The Frame 1 luminance was $49.2 \mathrm{~cd} / \mathrm{m}^{2}$ for one surface and $30.1 \mathrm{~cd} / \mathrm{m}^{2}$ for the other. The luminance of the lighter-than-background surface increased to $69.0 \mathrm{~cd} / \mathrm{m}^{2}$ or decreased to $43.4 \mathrm{~cd} / \mathrm{m}^{2}$. The luminance of the darker-than-background surface increased to $38.0 \mathrm{~cd} / \mathrm{m}^{2}$ or decreased to $1.1 \mathrm{~cd} / \mathrm{m}^{2}$. The BRLC was 1.1 for all these luminance changes. The left/right location of the light and dark surfaces and whether they carried the toward or away change were counterbalanced. There were four blocks of 120 order-randomized trials for each of the ICI ranges $(0-400$ and $0-120 \mathrm{msec})$, which were tested during separate sessions. Pretesting indicated that motion was not perceived for toward-then-toward or away-then-away changes.

Experiment 1C. Motion was specified by counterchanging texture contrast. The second-order stimuli were composed of a pair of square surfaces and a background composed of an equal number of randomly located, light and dark 1.4-min elements (average luminance $=26.1 \mathrm{~cd} / \mathrm{m}^{2}$ ). There were neither changes in location nor reversals in contrast polarity. The Frame 1 Michelson contrast of the elements composing both surfaces was 0.4 (luminance was 36.8 and $15.3 \mathrm{~cd} / \mathrm{m}^{2}$ for the light and dark elements). During Frame 2, the contrast of one surface increased to 1.0 (luminance values were 52.1 and $0.1 \mathrm{~cd} / \mathrm{m}^{2}$ ) or decreased to 0.2 (luminance values were 31.8 and $20.7 \mathrm{~cd} / \mathrm{m}^{2}$ ). The contrast of the other surface changed in the opposite direction during Frame 3. The Michelson contrast was 0.1 for the elements constituting the background (luminance values were 28.9 and $\left.23.2 \mathrm{~cd} / \mathrm{m}^{2}\right)$. There were four blocks of 168 order-randomized trials for each of the ICI ranges $(0-400$ and $0-120 \mathrm{msec}$ ).

Participants in all the experiments in this study were instructed to maintain attention midway between the two surfaces throughout the trial, and to avoid shifting their attention from one surface to the other. After each trial, they indicated whether or not they perceived motion and, if so, whether the motion was rightward or leftward. In addition to an author (O3), 4 naive participants were distributed across Experiments 1A, 1B, and 1C (3 in Experiments 1A and 1B, 2 in Experiment 1C).

\section{Results}

There was no systematic effect of motion direction, so the data for leftward and rightward motions were averaged, as were the data for the left/right positions of the lighter and darker than background squares in Experiment 1B. For all the participants in all three subexperiments, motion was perceived only when a toward change was accompanied by an away change. It always was in the counterchangespecified direction, regardless of whether the toward and away changes were simultaneous or sequential (Figure 3).

Motion was not based on sequential information. It was not perceived for sequences of away changes or sequences of toward changes, even though temporal parameters and luminance changes were the same as when there was counterchange. When perceived, motion was from the toward to the away change, even when the away change preceded the toward change. That is, when the two conflicted, motion was in the antisequential, counterchangespecified direction.

Finally, motion was perceived most often when toward and away changes were simultaneous, the frequency declining monotonically with increased ICI (more rapidly when the away change preceded the toward change than vice versa). Additional testing with a lower BRLC value confirmed for Experiment $1 \mathrm{~A}$ that this was not an artifact of ceiling effects. The frame preceding the toward and away changes in this experiment was very long $(2,000 \mathrm{msec})$, so optimal motion perception at an ICI of $0 \mathrm{msec}$ was consistent with the trade-off between frame duration and interstimulus interval that is described by Korte's fourth law for standard apparent motion: Longer frame durations result in optimal motion for smaller interstimulus intervals (Korte, 1915).

Motion energy. Although the Fourier space-time transformation of the generalized apparent motion stimulus in Figure 2A is complex, Figure 4A shows that it contains 


\section{Same Luminance Polarity (Experiment 1A)}

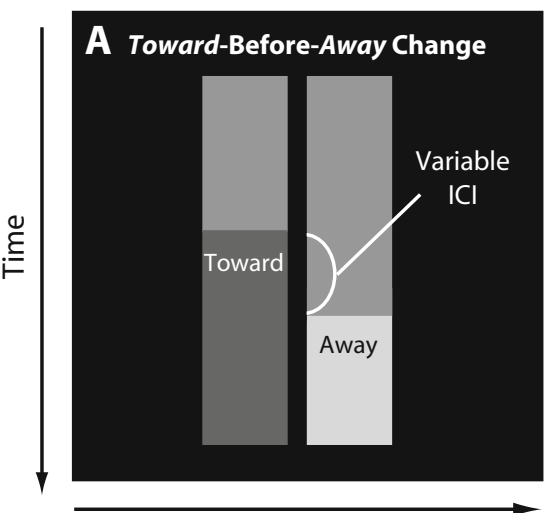

Spatial Position $(x)$

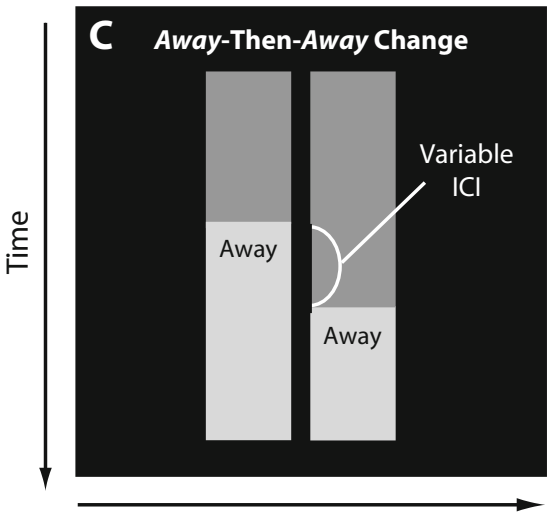

Spatial Position $(x)$
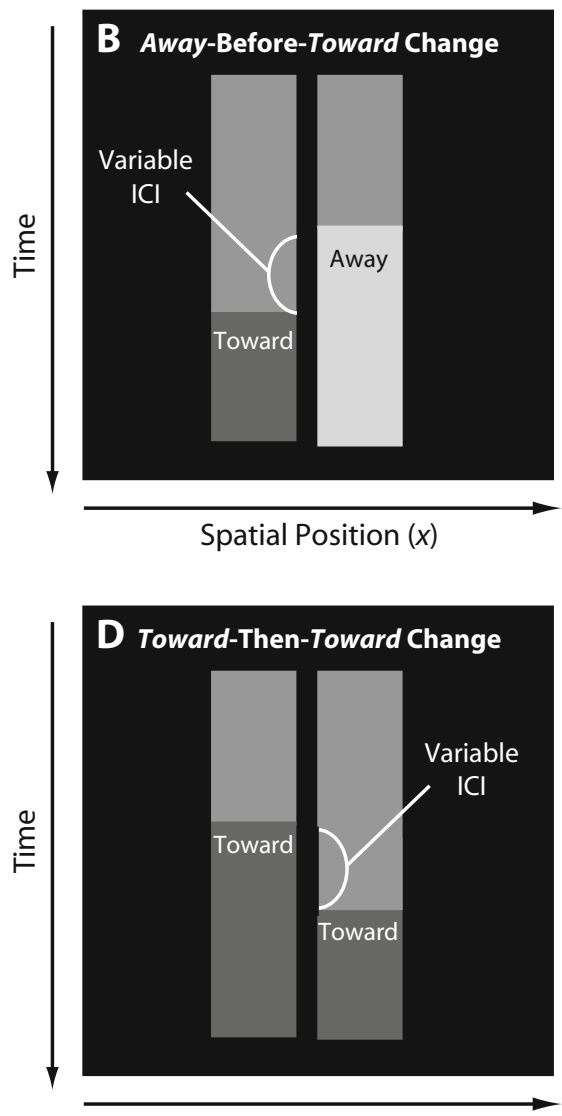

Spatial Position $(x)$

\section{Mixed Luminance Polarity (Experiment 1B)}
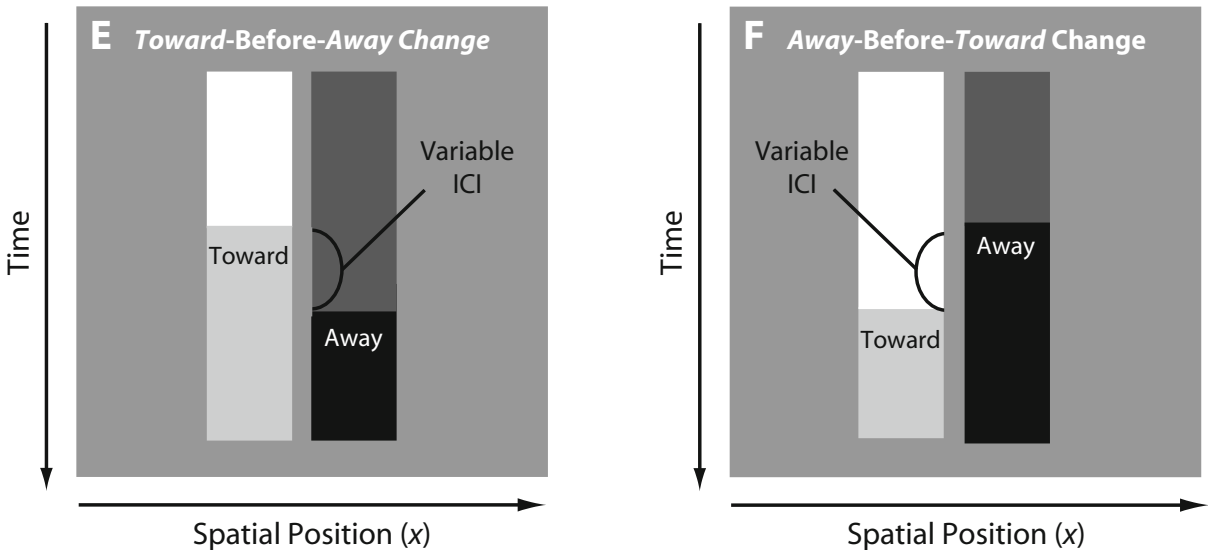

Figure 2. Graphical representations of the lighter-than-background stimuli in Experiment 1A: $(A, B)$ with counterchange, and $(C, D)$ without counterchange. $(E, F)$ Graphical representations of the stimuli with mixed luminance polarity in Experiment 1B. The ICI is the interchange interval.

oriented energy (the leftward orientation specifies rightward motion). ${ }^{4}$ The oriented motion energy is identical when the toward precedes the away change and vice versa. Could the detection of this motion energy be sufficient for the perception of generalized apparent motion? This possibility was evaluated by computationally implementing
Adelson and Bergen's (1985) motion energy model. It indicated that the detected motion energy is greater when the away change precedes the toward change. However, more motion was perceived in Experiment 1 when the toward change preceded the away change. Further evidence came from Experiment 1B, for which perceived apparent mo- 


\section{Experiment 1A \\ Same Luminance Polarity}
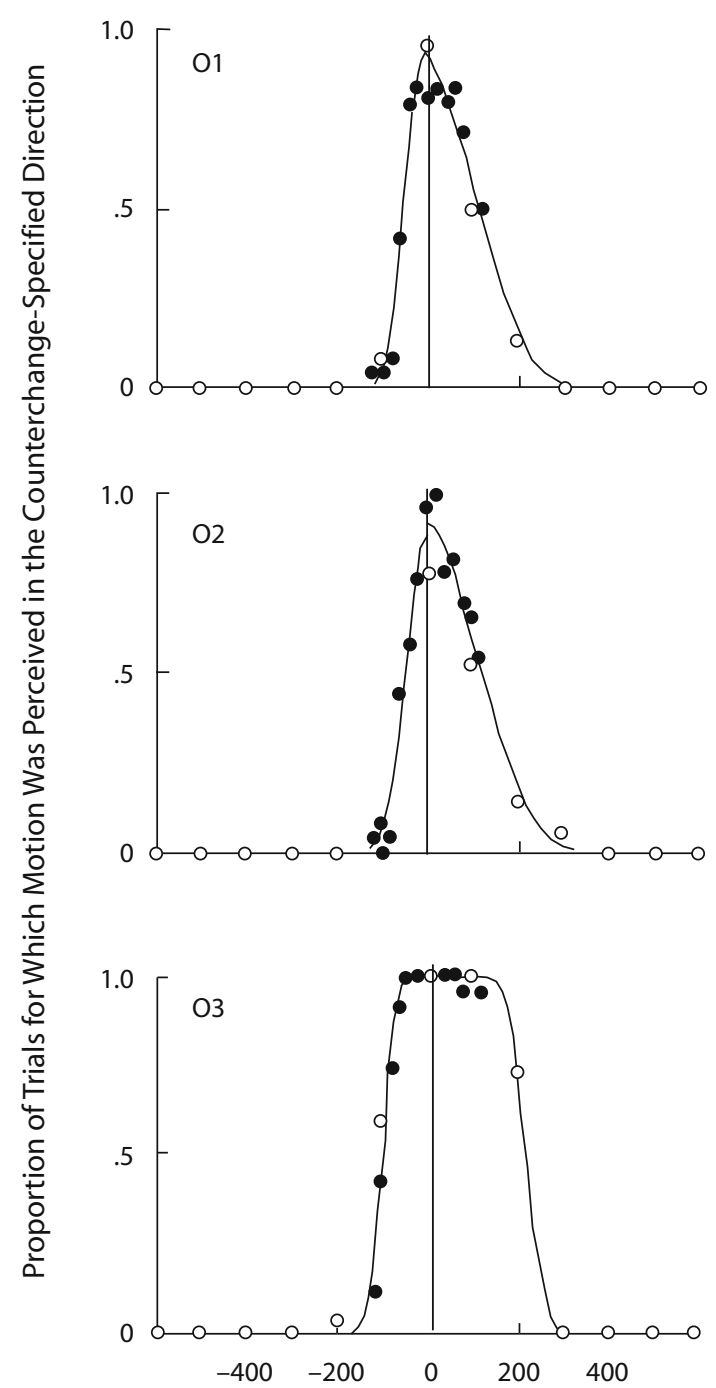

Experiment 1B

Mixed Luminance Polarity

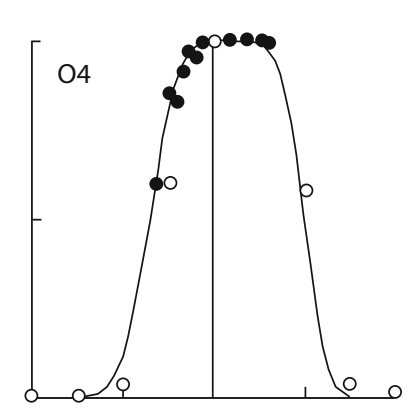

Experiment 1C Texture Contrast
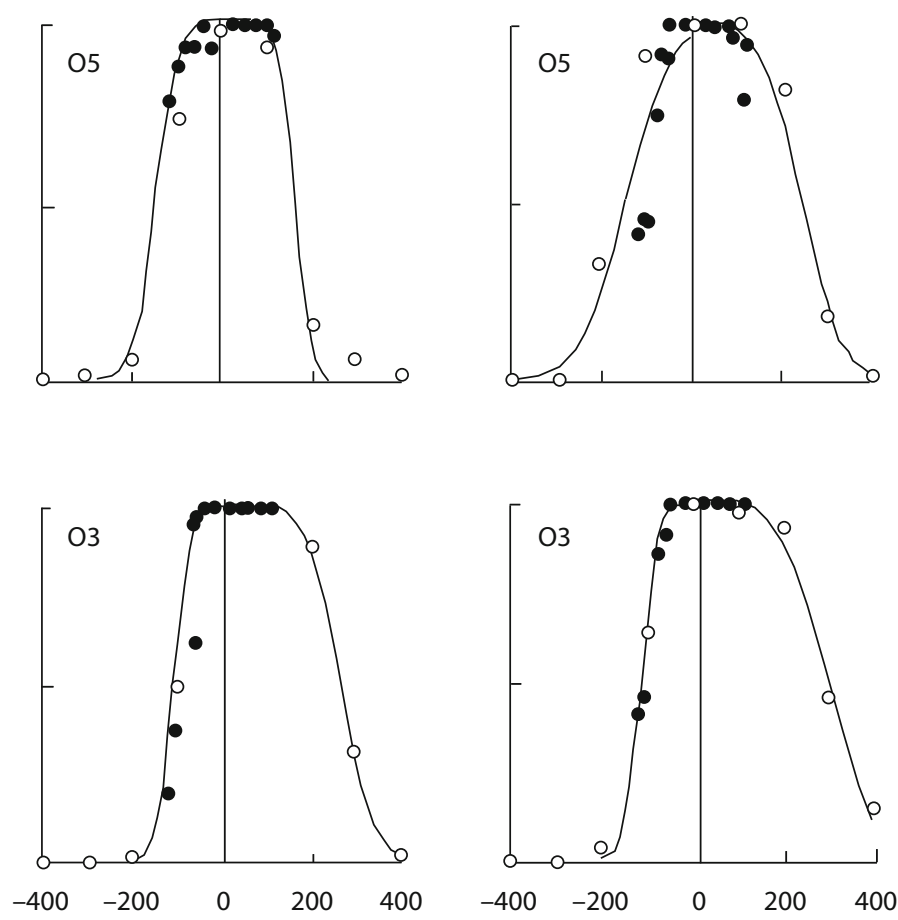

Interchange Interval (msec)

Figure 3. Results for Experiment 1A (all luminance values of the surfaces are greater than that of the background), Experiment 1B (one surface is lighter and the other is darker than the background), and Experiment $1 \mathrm{C}$ (all values of texture contrast for the surfaces are greater than the texture contrast of the background). In each case, the proportion of trials on which motion was perceived is graphed as a function of the interchange interval (ICI) for the toward-before-away condition (positive ICI values) and the awaybefore-toward condition (negative ICI values). Filled and open symbols denote data obtained from separate blocks of trials with different ranges of ICI values. Also included in each graph are best-fitting cumulative Gaussian functions, calculated separately for the toward-before-away and away-before-toward conditions. The results for the ICI $=0$ trials are included in both calculations. Motion never was reported for sequences of toward changes or sequences of away changes.

tion was opposite to the direction specified by the oriented motion energy. For example, leftward motion is specified by the rightward orientation of the motion energy diagram in Figure 4B. However, the perceived motion was in the rightward, counterchange-specified direction, for which there was no motion energy. Both of these instances are consistent with the perceived motion for generalized apparent motion stimuli being specified by counterchange rather than motion energy. ${ }^{5}$
Reverse-phi motion. Away-before-toward motion is similar to reverse-phi motion (Anstis \& Rogers, 1975) only in that its perceived direction is opposite to the temporal order of the visual events; motion begins at the location of the second event and ends at the location of the first event. Reverse-phi motion is in the direction specified by first-order motion energy. It occurs when multiple surfaces change their luminance polarity with successive displacements (lighter-than-background surfaces are re- 
Interchange Interval = 50 msec; Same Luminance Polarity

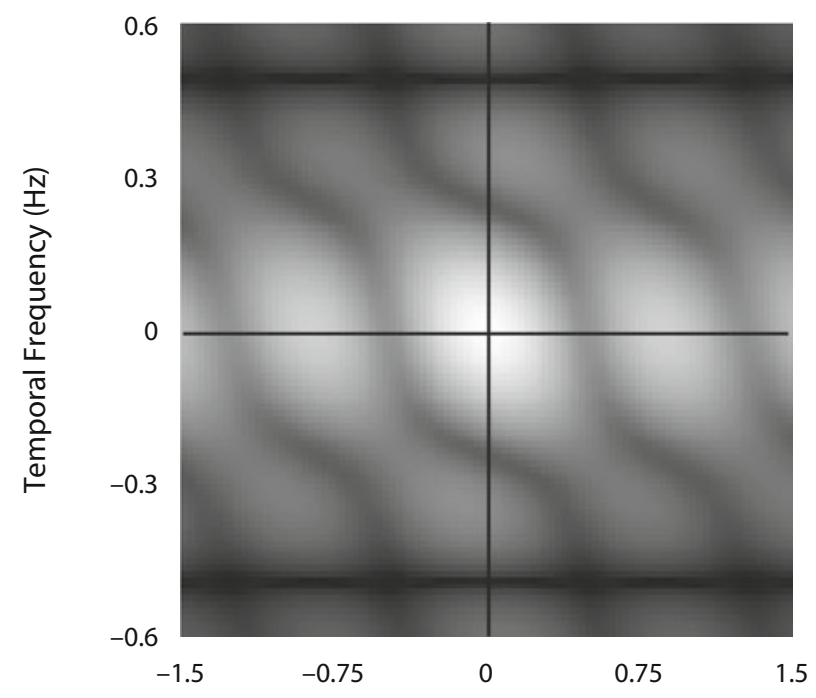

Spatial Frequency (cycles/deg)

B
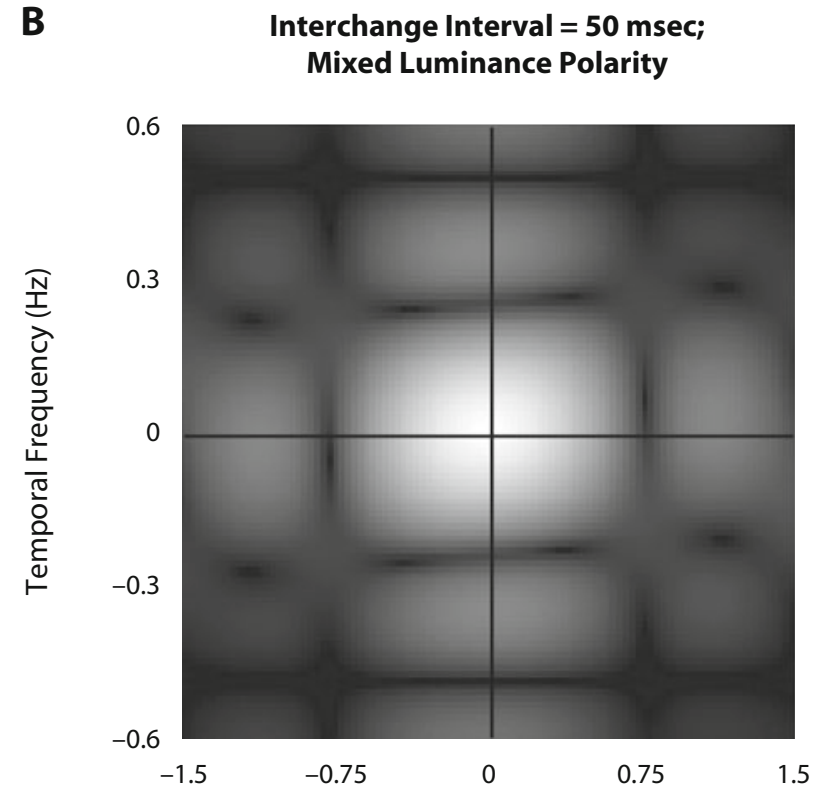

Spatial Frequency (cycles/deg)

Figure 4. (A) Fourier space-time transforms for the lighterthan-background stimuli in Experiment $1 \mathrm{~A}$ (graphical representations of the stimuli are in Figures $2 \mathrm{~A}$ and 2B). Rightward motion energy is indicated by the leftward orientation of the graph. The motion energy diagram is the same regardless of whether toward changes precede the away changes, or vice versa, by $50 \mathrm{msec}$. (B) Fourier space-time transforms for the mixed polarity stimuli in Experiment 1B (graphical representations of the stimuli are in Figures $2 \mathrm{E}$ and $2 \mathrm{~F}$ ). Leftward motion energy is indicated by the rightward orientation of the graph. The motion energy diagram again is the same, regardless of whether toward changes precede away changes, or vice versa, by 50 msec. placed by displaced darker-than-background surfaces) or when a single surface is partially displaced and there are gradual changes in luminance polarity.

The situation is different for away-before-toward motion. Reversals of luminance polarity are not required for the perception of antisequential motion; for example, both surfaces can be lighter or both darker than their background (Figure 2B). When there are reversals of luminance polarity, motion is not in the antisequential direction when toward changes precede away changes (Figure 2E), despite the presence of first-order motion energy specifying reverse-phi motion (Figure 4B). Antisequential motion is perceived only when the away change precedes the toward change, as in Figure 2F. If the antisequential motion in Experiment 1B were equivalent to reverse-phi motion, the perceived direction would have been opposite to the sequence of luminance changes, irrespective of the order of the toward and away changes (the oriented energy was the same in both cases).

\section{EXPERIMENT 2}

The purpose of this experiment was to establish that the evidence in the preceding experiment against sequencedetermined motion perception was not an artifact of coarse temporal sampling. That is, it might be argued that motion was perceived in the counterchange direction when the away change preceded the toward change because the temporal order of the stimulus changes could not be discerned, eliminating the availability of motion-specifying sequential information.

\section{Method}

In addition to the direction of the perceived motion, participants in this experiment reported the temporal order of the changes: whether the decrease in luminance at one location preceded the increase in luminance at the other location, or vice versa (there was no feedback and only enough practice to familiarize participants with the task). The away change preceded the toward change by $50 \mathrm{msec}$ for half the trials, and vice versa for the other half. Experiment $2 \mathrm{~A}$ was otherwise the same as Experiment 1A; likewise for Experiments 2B and $1 \mathrm{~B}$ and Experiments $2 \mathrm{C}$ and $1 \mathrm{C}$.

\section{Results}

As in Experiment 1, there were no systematic differences for rightward and leftward motion trials, so the data were averaged across motion direction (Figure 5). All the participants in all three subexperiments were able to resolve the temporal order of the toward and away changes (gray bars). The order was correctly reported for an average of $93.0 \%$ of the trials in which toward preceded away changes and for an average of $88.6 \%$ of the trials in which away preceded toward changes. The small decrease in accuracy when the away change came first was attributable to interference with judgments of temporal order from perceived motion being in the opposite direction.

Apparent motion was always perceived. As in Experiment 1 , it always started at the location of the toward change and ended at the location of the away change, even when the latter preceded the former (black bars in Figure 5). 
Experiment 2A: Same Luminance Polarity

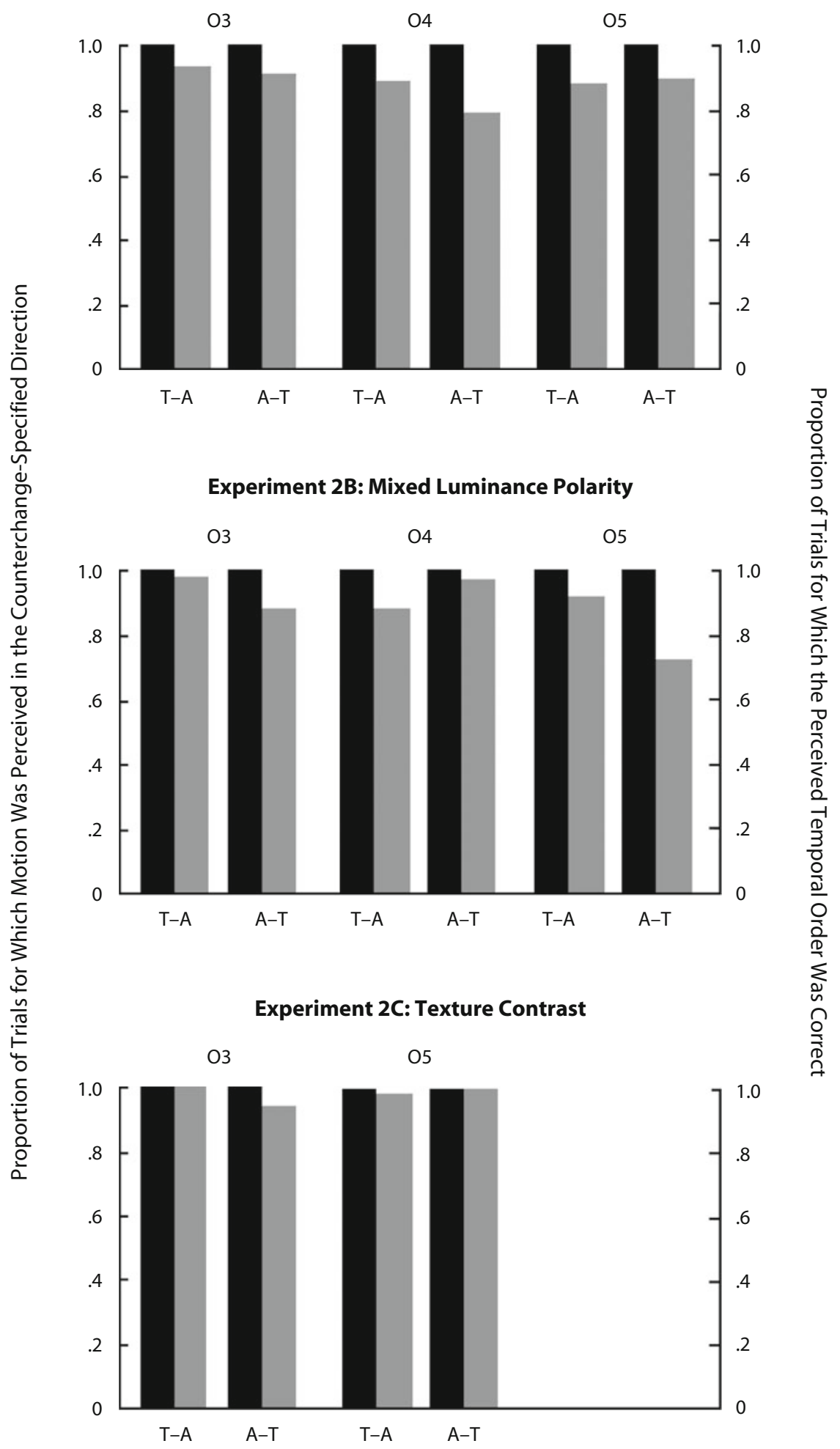

Figure 5. Results for Experiment 2. The proportion of trials for which motion was perceived in the counterchange-specified direction (black bars) and the proportion of trials for which temporal order was correctly identified (gray bars) for the toward-before-away ( $\mathrm{T}-\mathrm{A}$ ) and away-before-toward (A-T) conditions. 
Motion in the away-before-toward condition therefore was perceived in the counterchange-specified direction, even though participants could determine the correct temporal order of the luminance or contrast changes, information that would have been the basis for motion in the opposite direction if the perceived motion were determined by sequential order.

\section{Discussion}

The above experiments indicated that the perception of single-element apparent motion between two surfaces depends on the detection of toward/away contrast counterchange, even when the toward and away changes are presented sequentially (regardless of their order). In addition, motion was not perceived for sequences of away changes or sequences of toward changes, indicating that apparent motion perception does not require onset or offset asynchrony (see also Bischof \& Di Lollo, 1995). What about previous evidence that apparent motion perception increases with increased onset asynchrony (Kahneman \& Wolman, 1970; Kolers, 1964; Lakatos \& Shepard, 1997)? The essential difference between these earlier studies and Experiment 1 is that the stimuli in the earlier studies were composed of sequences of relatively brief flashes. This meant that onsets and offsets occurring at the same location were in close temporal proximity (in contrast, onsets and offsets were temporally isolated in
Experiments 1 and 2). In the experiments that follow, it is shown that earlier results attributed to onset or offset asynchrony are attributable instead to the detection of away changes (onsets) being adversely affected by immediately following toward changes (offsets) at the same location, and to the detection of toward changes (offsets) being adversely affected by immediately preceding away changes (onsets) at the same location. Such results would be consistent with processing units for detecting away and toward changes that are temporally biphasic: Units that are excited by changes in one direction are inhibited by changes in the opposite direction immediately preceding or immediately following.

\section{EXPERIMENT 3}

In this experiment, simultaneous toward/away counterchange at two surface locations was followed by the removal of the surface for which the away change had just occurred, creating consecutive away and toward changes for the same surface (Figure 6A). It was determined whether motion perception would be affected by the time between these events.

\section{Method}

The spatial dimensions of the stimuli and procedure were as in Experiment 1A. The luminance of both surfaces was $40.0 \mathrm{~cd} / \mathrm{m}^{2}$
A

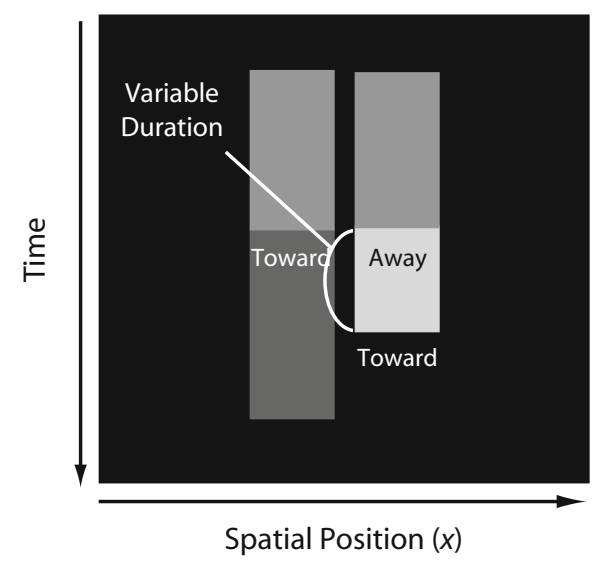

B Results

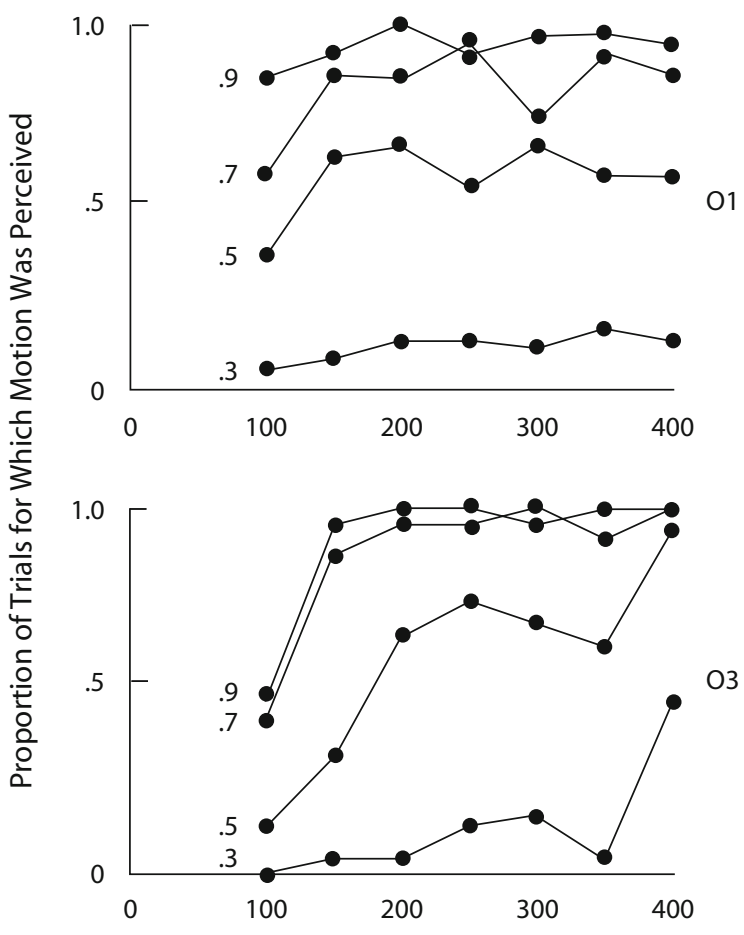

Duration Following Simultaneous Counterchange (msec)

Figure 6. (A) Graphical representation of the stimulus for Experiment 3. (B) The proportion of trials for which motion was perceived in the counterchange-specified direction for background-relative luminance change values ranging from 0.3 to 0.9 . 
during the 2,000-msec first frame. During the second frame, it increased for one surface to $43.5,51.5,62.0,75.5$, or $94.0 \mathrm{~cd} / \mathrm{m}^{2}$, and simultaneously, it decreased for the other surface to $36.7,31.3,26.8$, 22.8 , or $19.5 \mathrm{~cd} / \mathrm{m}^{2}$. These luminance changes resulted in BRLC values of $0.1,0.3,0.5,0.7$, and 0.9 , respectively (background luminance $=6.9 \mathrm{~cd} / \mathrm{m}^{2}$ ). The surfaces undergoing toward and away changes were equally often on the left and right, so counterchangespecified motion was equally often leftward and rightward. The surface for which there had been a toward change was removed $400 \mathrm{msec}$ after the simultaneous toward/away counterchange. The duration between the simultaneous toward/away counterchange and the removal of the surface for which an away change had just occurred (creating a toward change at that location) varied randomly from 100 to $400 \mathrm{msec}$. The orthogonal combination of seven durations, five BRLC values, and two motion directions resulted in 70 distinct trials, each of which was repeated three times within blocks of 210 trials (order-randomized in blocks of 70 trials). Four blocks of these trials were tested in a single session for 2 participants, both of whom had been tested in a preceding experiment.

\section{Results}

Irrespective of the BRLC value of the stimulus, motion perception was reduced when there was a relatively brief time interval between the simultaneous toward/away counterchange and the toward change occurring at the same location as the preceding away change (Figure 6B). This was consistent with away detectors being excited by away-from-background increases in contrast and inhibited by immediately following toward-background decreases in contrast at the same location, as would be expected if away detection were biphasic.

Although there were successive toward changes at the two spatial locations, it was shown in Experiment 1 that offset asynchrony is not sufficient for apparent motion perception.

\section{EXPERIMENT 4}

In this experiment, simultaneous toward/away counterchange at two surface locations was preceded by an away change at the location of a to-be-detected toward change, creating consecutive away and toward changes at that loca- tion (Figure 7A). It was determined whether motion perception would be affected by the time between these events.

\section{Method}

The spatial dimensions of the stimuli were as in the previous experiments. During the 2,000-msec first frame, the luminance was $2.0 \mathrm{~cd} / \mathrm{m}^{2}$ for one surface and $44.2 \mathrm{~cd} / \mathrm{m}^{2}$ for the other (background luminance $=1.1 \mathrm{~cd} / \mathrm{m}^{2}$ ). During Frame 2, the surface with the lower luminance increased in luminance to $44.2 \mathrm{~cd} / \mathrm{m}^{2}$, creating an away change. The duration of this frame varied from 25 to $375 \mathrm{msec}$. During Frame 3, the surface for which an away change had just occurred was removed, creating a toward change, and simultaneously, the surface at the other location increased in luminance from 44.2 to $68.3 \mathrm{~cd} / \mathrm{m}^{2}$, creating an away change. This surface was removed $400 \mathrm{msec}$ later. The orthogonal combination of eight durations preceding the simultaneous counterchange, two directions (left and right), and eight repetitions resulted in blocks of 128 trials (orderrandomized in blocks of 16 trials). Two new naive participants were tested on three blocks of trials during each of three testing sessions.

\section{Results}

Motion perception was reduced when there was a relatively brief time interval between the simultaneous toward/ away counterchange and the preceding away change occurring at the same location as the toward change (Figure 7B). This was consistent with toward detectors being excited by toward-background decreases in contrast and inhibited by immediately preceding away-from-background increases in contrast at the same location, as would be expected if toward detection were biphasic.

Although there were successive away changes at the two spatial locations, it was shown in Experiment 1 that onset asynchrony is not sufficient for apparent motion perception.

\section{DISCUSSION}

\section{A Mechanism for Detecting Counterchange- Specified Motion}

The experimental results reported in this article are consistent with a directionally selective mechanism for
A

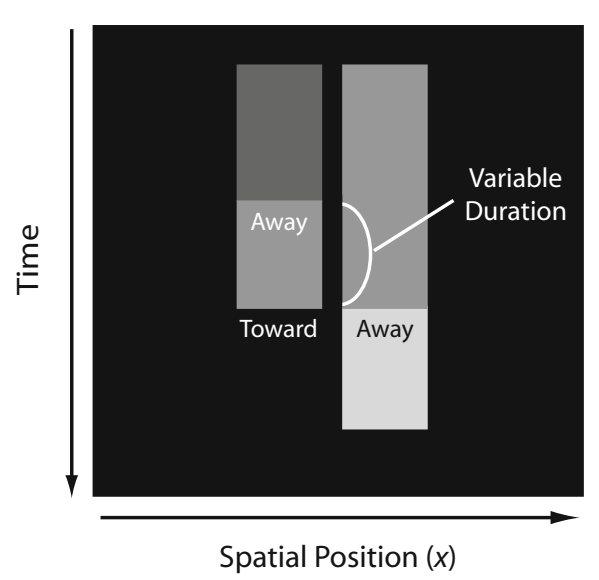

B

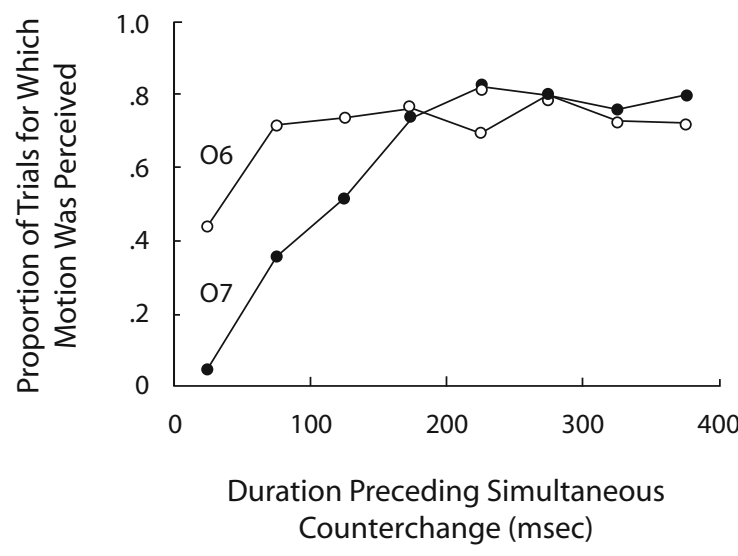

Figure 7. (A) Graphical representation of the stimulus in Experiment 4. (B) The proportion of trials for which motion was perceived in the counterchange-specified direction. 
the detection of counterchange-specified motion proposed by Hock et al. (2002) and Gilroy and Hock (2004a). The mechanism is composed of two spatially separated subunits, one responding transiently to toward-background changes (signaling the start of the motion path) and the other responding transiently to away-from-background changes (signaling the end of the motion path). Motion, which is given by the product of the subunit responses (Gilroy \& Hock, 2004b), is signified when there is sufficient temporal overlap of these responses, and the direction of the perceived motion is determined by the relative spatial location of the subunits responding to the toward and away changes. The absence of an explicit delay mechanism in the model reflects the existence of substantial experimental evidence indicating that the detection of sequential stimulus information is neither necessary nor sufficient for the perception of apparent motion and by computational evidence that delay can be an emergent property of the transient detection of stimulus changes (Hock, Schöner, \& Gilroy, 2009).

The proposed mechanism was motivated by evidence that apparent motion between two spatial locations is perceived only when there is a toward-background change in luminance and/or texture contrast at the location of one surface and an away-from-background change in luminance and/or texture contrast at the location of the other surface (Gilroy \& Hock, 2004a; Hock \& Gilroy, 2005; Hock et al., 2002). Evidence that the toward and away changes at different spatial locations must occur relatively proximally in time (Experiment 1 of the present article) is consistent with each subunit's responding transiently to these changes. Transient responses have a finite time course of activation, so it is possible for a pair of subunit responses to overlap in time, even when the stimulus changes giving rise to those responses do not occur simultaneously; the greater the time between the toward and away changes, the less the overlap and, therefore, the less frequently motion is perceived. The overlap can occur even when the away change precedes the toward change, leading to motion's being perceived in the antisequential direction (Experiments 1 and 2).

The results of Experiments 3 and 4 were consistent with the transient responses for each subunit being generated by biphasic detectors that temporally integrate an initially excitatory response to the input with a subsequent inhibitory response. The excitatory and inhibitory responses balance out when the input is constant and produce a transient response when the input changes. The biphasic toward subunit would respond with excitation to decreases in activation and with inhibition to increases in activation, whereas the biphasic away subunit would respond with excitation to increases in activation and with inhibition to decreases in activation. When a to-be-detected away change is immediately followed by the removal of the stimulus (a toward change at the same location), the likelihood of motion being perceived is reduced because the growth in excitation produced by the away change is cut off before reaching its maximum (Experiment 3). When a to-be-detected toward change is immediately preceded by an away change at the same location, the likelihood of motion's being perceived is reduced because there is insufficient time for recovery from the inhibition produced by the preceding away change (Experiment 4). However, brief intervals between away and toward changes at the same location (i.e., brief frame durations) can result in the consistent perception of apparent motion, if sufficient time for recovery from biphasic inhibition is provided by an interstimulus interval between the luminance changes at the two locations. Korte's fourth law, which describes the trade-off between frame duration and interstimulus interval, has been demonstrated computationally by Hock et al. (2009).

The experiments reported in the present article have focused on the temporal dynamics of counterchange detection for the perception of apparent motion. The results of other experiments (Hock \& Nichols, in press) have indicated that edge detectors, as well as detectors of surface/background contrast (center/surround units), provide the spatial input to the toward and away subunits of the counterchange motion detector. It has been shown by Hock et al. (2009) that the counterchange model, with these filters providing spatial input, can potentially account for other motion phenomena. These include Gabor patches with drifting sine gratings, displaced sections of random cinematograms that create short-range motion (Braddick, 1974; Lappin \& Bell, 1972), the line motion illusion (Hock \& Nichols, in press; Shimojo, Miyauchi, \& Hikosaka, 1997), and, most importantly, continuous object motion.

\section{Continuous Object Motion}

An object moving continuously through space creates discontinuous changes at its leading and trailing edges. For example, when a light object moves across a dark background, there are discontinuous increases in luminance at its leading edge and discontinuous decreases in luminance at its trailing edge (Figure 8A). Although apparent motion is a temporally sampled version of continuous motion (Adelson \& Bergen, 1985), the perception of continuous object motion is not directly analogous to the perception of apparent motion. If it were, motion would be perceived across the object, from its back (where luminance contrast decreases) to its front (where luminance and luminance contrast increase). This, however, does not occur in reality. If the perception of continuous object motion were based on the detection of counterchange, motion would be established independently at the leading and trailing edges of the object by the detection of decreases in edge contrast accompanied by increases in surface/background contrast.

This is illustrated in Figure $8 \mathrm{~B}$, where it can be seen that the edge filter, which is activated when the leading boundary of the moving object enters its excitatory lobe, has its activation decrease a moment later when the leading boundary of the object enters its inhibitory lobe. Soon afterward, the leading boundary of the moving object enters the excitatory center of the center/surround filter, increasing its activation. This combination of decreased activation for the edge filter and increased activation for the center/surround filter results in counterchange- 

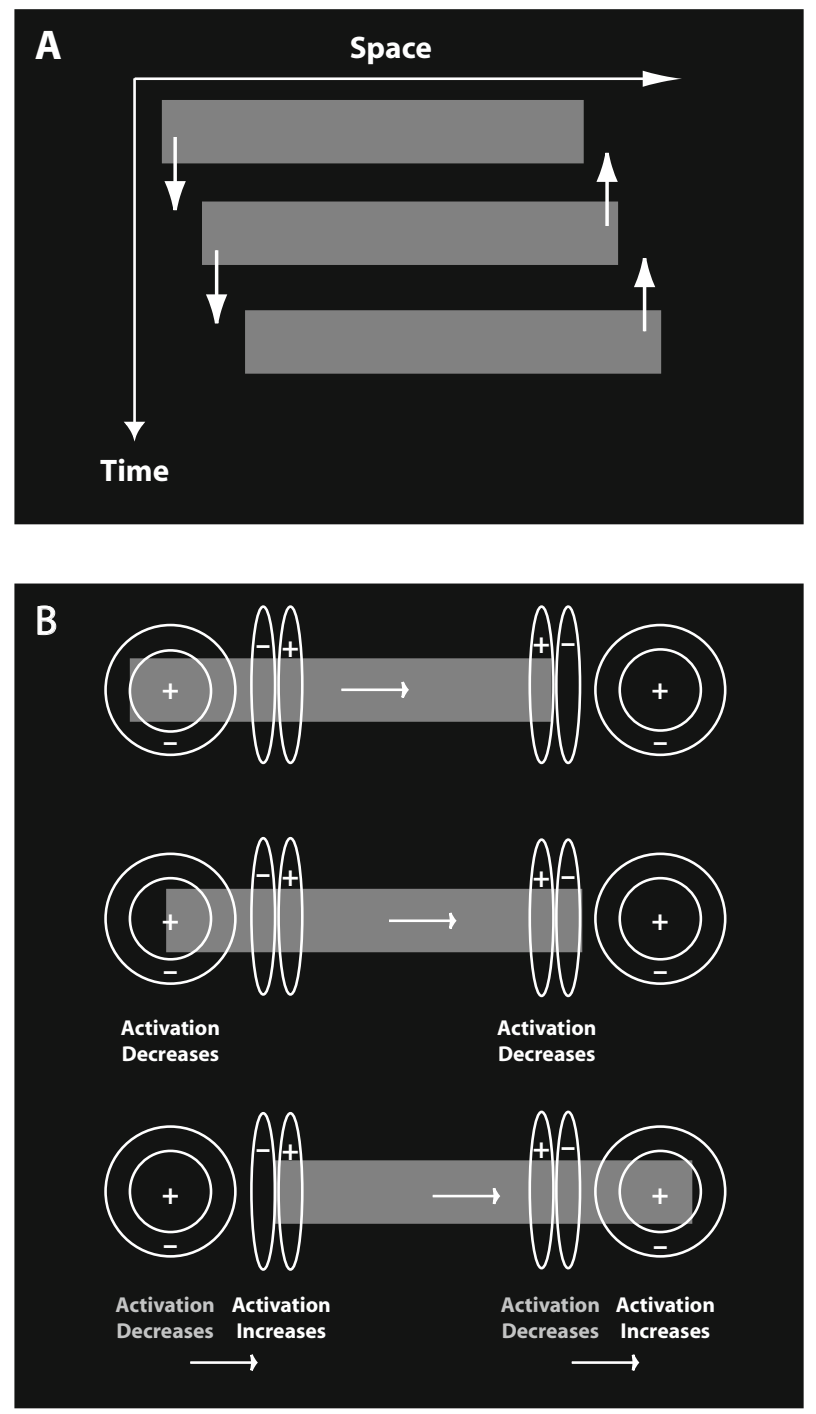

Figure 8. (A) Temporal discontinuities at the leading and trailing edges of a continuously moving object. (B) Schematic of the counterchange model as applied to the perception of continuous motion of an object.

specified rightward motion at the object's leading edge. Counterchange-specified rightward motion is similarly signaled at the trailing edge of the moving object by the combination of decreased center/surround activation and increased edge filter activation. Further research will test this application of the counterchange principle to the perception of continuous object motion.

\section{CONCLUSION}

For standard apparent motion, a surface is discontinuously displaced from one location to another, so it is possible that the perception of motion depends on the detection of sequential changes in location rather than counterchange (luminance decreases at the initial location of the surface and increases at its newly occupied location). The specification of motion by sequential changes in location is not possible for the generalized apparent motion stimuli studied in this article (both locations are always occupied by a surface), and it is further shown that it is not specified by sequential changes in luminance (e.g., by a succession of onsets or a succession of offsets). Instead of sequence, apparent motion between two spatial locations is perceived via the detection of counterchange, from the location of the toward to the location of the away change, even when the changes occur in reverse temporal order. The results thus constitute evidence that sequential stimulus information does not provide bottom-up input that is sufficient for the perception of apparent motion. (It could, however, provide the basis for top-down attentional mechanisms to create motion percepts; Cavanagh, 1992.) Instead, the results are consistent with the existence of a directionally selective mechanism for the detection of counterchangespecified motion, a mechanism that is a likely basis for third-order motion perception (Sperling \& Lu, 1998). A computational model of this mechanism, which is based on the biphasic detection of toward and away changes, has been described in detail by Hock et al. (2009).

\section{AUTHOR NOTE}

This work was partially supported by NIH Grant EY07760. We thank Gregor Schöner and Joe Lappin for comments on an earlier version of the article. We also thank David Nichols for the computational implementation of Adelson and Bergen's (1985) motion energy model. Direct correspondence to H. S. Hock, Department of Psychology, Florida Atlantic University, Boca Raton, FL 33431 (e-mail: hockhs@,fau.edu).

\section{REFERENCES}

Adelson, E. H., \& Bergen, J. R. (1985). Spatiotemporal energy models for the perception of motion. Journal of the Optical Society of America A, 2, 284-299.

Anstis, S. M., \& MAther, G. (1985). Effects of luminance and contrast on direction of ambiguous apparent motion. Perception, 14, 167-179.

ANSTIS, S. M., \& Rogers, B. J. (1975). Illusory reversal of visual depth and movement during changes of contrast. Vision Research, 15, 957-961.

Bischof, W. F., \& Di Lollo, V. (1995). Motion and metacontrast with simultaneous onset of stimuli. Journal of the Optical Society of America A, 12, 1623-1636.

BRADDICK, O. J. (1974). A short-range process in apparent motion. $\mathrm{Vi}$ sion Research, 14, 519-527.

Burt, P., \& Sperling, G. (1981). Time, distance, and feature trade-offs in visual apparent motion. Psychological Review, 88, 171-195.

Cavanagh, P. (1992). Attention-based motion perception. Science, 257, 1563-1565.

Chubb, C., \& Sperling, G. (1989). Two motion perception mechanisms revealed through distance-driven reversal of apparent motion. Proceedings of the National Academy of Sciences, 86, 2985-2989.

ExNER, S. (1875). Über das Sehen von Bewegungen und die Theorie des Zusammengesetzten Auges [On motion perception and the theory of compound eyes]. Sitzungsberichte der Kaiserlichen Akademie der Wissenschaften, 72, 156-190.

Gibson, J. J. (1968). What gives rise to the perception of motion? Psychological Review, 75, 335-346.

Gilroy, L. A., \& Hock, H. S. (2004a). Detection of counter-changing contrast: Second-order apparent motion without postrectification motion-energy analysis or salience mapping/feature tracking. Journal of Experimental Psychology: Human Perception \& Performance, 30, 137-150.

Gilroy, L. A., \& Hock, H. S. (2004b). Multiplicative nonlinearity in the perception of apparent motion. Vision Research, 44, 2001-2007. 
Hock, H. S., \& Gilroy, L. (2005). A common mechanism for the perception of first-order and second-order apparent motion. Vision Research, 45, 661-675.

Hock, H. S., Gilroy, L. A., \& Harnett, G. (2002). Counter-changing luminance: A non-Fourier, non-attentional basis for the perception of single-element apparent motion. Journal of Experimental Psychology: Human Perception \& Performance, 28, 93-112.

Hock, H. S., Kogan, K., \& Espinoza, J. K. (1997). Dynamic, statedependent thresholds for the perception of single-element apparent motion: Bistability from local cooperativity. Perception \& Psychophysics, 59, 1077-1088.

Hock, H. S., \& NichOLs, D. F. (2004). Separating the edge-based detection of object motion from the objectless detection of motion energy [Abstract]. Perception, 33, 35.

Hock, H. S., \& Nichols, D. F. (in press). The line motion illusion: The detection of counterchanging edge and surface contrast. Journal of Experimental Psychology: Human Perception \& Performance.

Hock, H. S., Schöner, G., \& GiLroy, L. A. (2009). A counterchange mechanism for the perception of motion. Acta Psychologica, 132, $1-21$

Johansson, G. (1950). Configurations in event perception. Uppsala, Sweden: Almqvist \& Wiksell

Kahneman, D., \& Wolman, R. E. (1970). Stroboscopic motion: Effects of duration and interval. Perception \& Psychophysics, 8, 161-164.

Kolers, P. A. (1964). The illusion of movement. Scientific American, 211, 98-106.

Korte, A. (1915). Kinematoskopische Untersuchungen [Kinematoscopic investigations]. Zeitschrift für Psychologie, 72, 194-296.

LaKatos, S., \& ShePard, R. N. (1997). Constraints common to apparent motion in visual, tactile, and auditory space. Journal of Experimental Psychology: Human Perception \& Performance, 23, 1050-1060.

Lappin, J. S., \& Bell, H. H. (1972). Perceptual differentiation of sequential visual patterns. Perception \& Psychophysics, 12, 129-134.

Lappin, J. S., Tadin, D., \& WhitTier, E. J. (2002). Visual coherence of moving and stationary image changes. Vision Research, 42, 1523-1534.

MatheR, G. (1984). Luminance change generates apparent movement: Implications for models of directional specificity in the human visual system. Vision Research, 24, 1399-1405.

Mather, G., \& Anstis, S. (1995). Second-order texture contrast resolves ambiguous apparent motion. Perception, 24, 1373-1382.

Mikami, A. (1992). Spatiotemporal characteristics of direction-selective neurons in the middle temporal visual area of the macaque monkeys. Experimental Brain Research, 90, 40-46.

REICHARDT, W. (1961). Autocorrelation, a principle for the evaluation of sensory information by the central nervous system. In W. A. Rosenblith (Ed.), Sensory communication (pp. 303-317). New York: Wiley.

Shapiro, A. G., D’Antona, A. D., Charles, J. P., Belano, L. A., Smith, J. B., \& Shear-Heyman, M. (2004). Induced contrast asynchronies. Journal of Vision, 4, 459-468.

Shimojo, S., Miyauchi, S., \& Hikosaka, O. (1997). Visual motion sensation yielded by non-visually driven attention. Vision Research, 37, 1575-1580.

SPERLING, G., \& Lu, Z.-L. (1998). A systems analysis of visual motion perception. In T. Watanabe (Ed.), High-level motion processing: Computational, neurobiological, and psychophysical perspectives (pp. 154-183). Cambridge, MA: MIT Press.

Watson, A. B. (1986). Apparent motion occurs only between similar spatial frequencies. Vision Research, 26, 1727-1730.

Wertheimer, M. (1912). Experimentelle Studien über das Sehen von Bewegung [Experimental studies on the visual perception of motion]. Zeitschrift für Psychologie und Physiologie der Sinnesorgane, 61, 161-265.

\section{NOTES}

1. Both Hock, Gilroy, and Harnett (2002) and Shapiro et al. (2004) have created stimuli for which first-order luminance changes and second-order contrast changes are simultaneously present. The stimuli in both studies were composed of two surfaces, one with a lighter surround and the other with a darker surround. When the luminance of the surfaces simultaneously increased, the surface contrast with the lighter surround decreased and the surface contrast with the darker surround increased (i.e., synchronous luminance changes co-occurred with asynchronous contrast changes). Shapiro et al.'s observers could track both the first-order luminance changes and the second-order contrast changes for each surface. While keeping their attention fixed midway between the surfaces, Hock et al.'s (2002) observers could perceive motion between the surfaces on the basis of the asynchronous contrast changes (second-order counterchange). When Hock et al.'s (2002) contrast changes were synchronous and their luminance changes asynchronous, motion was perceived, although weakly, on the basis of first-order luminance counterchange. Although their aims were different, both studies provided evidence for the simultaneous availability of first-order luminance information and second-order contrast information.

2. It might be argued that motion perception for the stimulus in Figure $1 \mathrm{~B}$ is based on sequential changes in the location of the lighter, higher contrast surface (it shifts to the right) competing with sequential changes in the location of the darker, lower contrast surface (it shifts to the left). However, similar results are obtained for stimuli like the one in Figure 1C. Opposing sequential changes are not possible for this stimulus, because both surfaces are identical prior to the toward/away counterchange. The stimulus configuration in Figure 1C is the basis for the experiments in this article.

3. Afterimages were not produced by the luminance decrements (toward changes $)$, perhaps because the surfaces were very small $(12 \times$ $12 \mathrm{~min}$ ). If such afterimages had occurred, they could have produced the equivalent of an away change for the surface that could have created counterchange-specified motion were it paired with a toward change for another surface.

4. The Fourier space-time transformation for a sequence of toward and away changes combines motion energy created independently by the toward change for one frame-to-frame transition and the away change for the other frame-to-frame transition. Although the two motion energy components differ in magnitude, their sum is the same when the toward precedes the away change, and vice versa. Motion is not perceived when there is only a toward change for one surface (the first transition), or when there is only an away change for one surface (the second transition), so the possibility that single-element apparent motion perception is based on motion energy extraction is viable only when the two motion energy components are combined.

5. Oppositely signed changes in background-relative luminance at two spatial locations are sufficient for the detection of counterchangespecified motion. In contrast, motion energy detection may require luminance changes at three or more spatial locations, and differs as well with respect to its preference for high-speed motion (Hock \& Nichols, 2004). These differences between counterchange and motion energy detection might have their neural counterpart in directionally selective neurons in area MT. Luminance changes at two spatial locations are sufficient for some MT neurons to exhibit directional selectivity. However, directional selectivity for other MT neurons requires luminance changes at three or more spatial locations (Mikami, 1992).

(Manuscript received October 8, 2008; revision accepted for publication April 9, 2009.) 